\title{
Video Article \\ Enhanced Sample Multiplexing of Tissues Using Combined Precursor Isotopic Labeling and Isobaric Tagging (cPILOT)
}

\author{
Christina D. King ${ }^{1}$, Joseph D. Dudenhoeffer ${ }^{1}$, Liqing $\mathrm{Gu}^{2}$, Adam R. Evans ${ }^{3}$, Renã A. S. Robinson ${ }^{1}$ \\ ${ }^{1}$ Department of Chemistry, University of Pittsburgh \\ ${ }^{2}$ SGS North America Inc. \\ ${ }^{3}$ Large Molecule Analytical Development, Pharmaceutical Development \& Manufacturing Science, Janssen Research and Development
}

Correspondence to: Renã A. S. Robinson at rena@pitt.edu

URL: https://www.jove.com/video/55406

DOI: doi: $10.3791 / 55406$

Keywords: Biochemistry, Issue 123, Quantitative proteomics, CPILOT, TMT, isotopic labeling and isobaric tagging, multiplexing, tissues

Date Published: 5/1/2017

Citation: King, C.D., Dudenhoeffer, J.D., Gu, L., Evans, A.R., Robinson, R.A. Enhanced Sample Multiplexing of Tissues Using Combined Precursor Isotopic Labeling and Isobaric Tagging (cPILOT). J. Vis. Exp. (123), e55406, doi:10.3791/55406 (2017).

\section{Abstract}

There is an increasing demand to analyze many biological samples for disease understanding and biomarker discovery. Quantitative proteomics strategies that allow simultaneous measurement of multiple samples have become widespread and greatly reduce experimental costs and times. Our laboratory developed a technique called combined precursor isotopic labeling and isobaric tagging (cPILOT), which enhances sample multiplexing of traditional isotopic labeling or isobaric tagging approaches. Global cPILOT can be applied to samples originating from cells, tissues, bodily fluids, or whole organisms and gives information on relative protein abundances across different sample conditions. cPILOT works by 1) using low $\mathrm{pH}$ buffer conditions to selectively dimethylate peptide $\mathrm{N}$-termini and 2) using high pH buffer conditions to label primary amines of lysine residues with commercially-available isobaric reagents (see Table of Materials/Reagents). The degree of sample multiplexing available is dependent on the number of precursor labels used and the isobaric tagging reagent. Here, we present a 12-plex analysis using light and heavy dimethylation combined with six-plex isobaric reagents to analyze 12 samples from mouse tissues in a single analysis. Enhanced multiplexing is helpful for reducing experimental time and cost and more importantly, allowing comparison across many sample conditions (biological replicates, disease stage, drug treatments, genotypes, or longitudinal time-points) with less experimental bias and error. In this work, the global cPILOT approach is used to analyze brain, heart, and liver tissues across biological replicates from an Alzheimer's disease mouse model and wild-type controls. Global cPILOT can be applied to study other biological processes and adapted to increase sample multiplexing to greater than 20 samples.

\section{Video Link}

The video component of this article can be found at https://www.jove.com/video/55406/

\section{Introduction}

Proteomics often involves the analysis of many samples used to better understand disease processes, enzyme kinetics, post-translational modifications, response to environmental stimuli, response to therapeutic treatments, biomarker discovery, or drug mechanisms. Quantitative methods can be employed to measure relative differences in protein levels across the samples and can be label-free or involve isotopic labeling (metabolic, chemical, or enzymatic). Stable isotope labeling methods have grown in popularity because they allow many samples to be analyzed simultaneously and are suitable for samples from different cells, tissues, bodily fluids, or whole organisms. Isotope labeling methods $\mathbf{s}^{1,2,3,4,5,6,7}$ increase experimental throughput, while reducing acquisition time, costs, and experimental error. These methods use precursor mass spectra to measure relative abundances of proteins from peptide peaks. In contrast, isobaric tagging reagents ${ }^{8,9,10}$ generate reporter ions that are either detected in $\mathrm{MS} / \mathrm{MS}$ or $\mathrm{MS}^{311}$ spectra and these peaks are used to report on relative abundances of proteins.

The current state-of-the-art in proteomics multiplexing is either a 10-plex ${ }^{12}$ or 12-plex isobaric tag analysis ${ }^{13}$. Enhanced sample multiplexing (i.e. $>10$ samples) methods have been developed by our laboratory for tissues ${ }^{14,15,16,17}$, and by others for the analysis of cells ${ }^{18,19,20}$, tissues 21 , or targeted peptides ${ }^{22}$. We developed an enhanced multiplexing technique called combined precursor isotopic labeling with isobaric tagging (cPILOT). Global cPILOT is useful for getting information about the relative concentrations of all proteins across different sample conditions $(\geq 12)^{14}$. Figure 1 shows a general cPILOT workflow. Tryptic or Lys-C peptides are selectively labeled at the N-terminus with dimethylation using low $\mathrm{pH}^{2}$ and at lysine residues with 6-plex reagents using high $\mathrm{pH}$. This strategy doubles the number of samples that can be analyzed with isobaric reagents which helps to reduce experimental costs and additionally, reduces experimental steps and time.

CPILOT is flexible as we have developed other methods to study oxidative post-translational modifications, including 3-nitrotyrosine-modified proteins ${ }^{14}$ and cysteine containing peptides with S-nitrosylation (oxcyscPILOT) ${ }^{23}$. We have also developed an amino acid selective approach, cysteine cPILOT (cyscPILOT) ${ }^{17}$. MS $^{3}$ acquisition with a top-ion ${ }^{11}$ or selective- $y_{1}$-ion method ${ }^{15}$ can help reduce reporter ion interference and improve quantitative accuracy of CPILOT. The use of $\mathrm{MS}^{3}$ in the acquisition method requires a high-resolution instrument with an orbitrap mass analyzer although low resolution ion trap instruments may also work ${ }^{24}$. 
Previously, cPILOT has been used to study liver proteins ${ }^{16}$ from an Alzheimer's disease mouse model. Here, we describe how to perform global CPILOT analysis using brain, heart, and liver homogenates to study the role of the periphery in Alzheimer's disease. This experiment incorporates biological replication. Because of the versatility of cPILOT, interested users can use the technique to study other tissues for a range of biological problems and systems.

\section{Protocol}

Ethics Statement: Mice were purchased from an independent, non-profit biomedical research institution and housed in the Division of Laboratory Animal Resources at the University of Pittsburgh. All animal protocols were approved by the Institutional Animal Care and Use Committee at the University of Pittsburgh.

\section{Protein Extraction and Generation of Peptides for Chemical-tagging}

1. Extract protein from tissue, cells, or bodily fluids.

1. Homogenize $60-90 \mathrm{mg}$ of tissue (e.g. brain, heart, and liver) in phosphate buffer saline (1x PBS) with $8 \mathrm{M}$ urea (500 $\mu \mathrm{L})$ using a mechanical homogenizer. Protease or phosphatase inhibitors may be added to the buffer if necessary. In this project, they were not used. Use the following parameters for the homogenizer: lysing matrix A beads, $4 \mathrm{~m} / \mathrm{s}, 20 \mathrm{~s}$. Heart tissue may require up to 9 cycles of homogenization.

NOTE: Protease or phosphatase inhibitors may be added to the buffer if necessary. They were not used in this study.

1. Remove tissue homogenate from the lysing tube and transfer to a micro-centrifuge tube. Rinse lysing tubes with 100-500 $\mu \mathrm{L}$ of PBS with $8 \mathrm{M}$ urea and combine the rinse solution with tissue homogenate.

2. Centrifuge the homogenized tissues $\left(9,447 \times \mathrm{g}, 4^{\circ} \mathrm{C}, 15 \mathrm{~min}\right)$ and collect the supernatant.

2. Determine the protein concentration using a bicinchoninic acid (BCA) assay according to manufacturer's instructions. NOTE: Further dilution with buffer may be necessary if the protein concentration is too high. The resulting concentrations for samples from these tissues were in between $6-13 \mu \mathrm{g} / \mu \mathrm{L}$.

1. Optional: Add an internal quality control standard (e.g. bovine alpha casein or other exogenous protein) with the ratio $1 \mu \mathrm{g}$ standard: $100 \mu \mathrm{g}$ protein sample.

\section{Sample Digestion}

1. Add $100 \mu \mathrm{g}\left(100 \times 10^{-6} \mathrm{~g}\right)$ of protein from each sample to individually labeled micro-centrifuge tubes. The volume is dependent on the protein concentration (see step 1.2).

2. Add dithiothreitol (DTT) (40:1 reagent to sample mol ratio) to each sample. Incubate at $37{ }^{\circ} \mathrm{C}$ for $2 \mathrm{~h}$. The calculation for the molar ratio is based off the protein mass of bovine serum albumin (BSA), which is $66.5 \times 10^{3} \mathrm{~g} / \mathrm{mol}$.

3. Add iodoacetamide (IAM) (80:1 reagent to sample mol ratio) to each sample. Incubate on ice in the dark for $2 \mathrm{~h}$. This reaction is performed on ice for $2 \mathrm{~h}$ to prevent side reactions.

4. Add L-cysteine (40:1 reagent to sample mol ratio) to each sample. Incubate at room temperature for $30 \mathrm{~min}$.

5. Add $20 \mathrm{mM}$ Tris buffer with $10 \mathrm{mM} \mathrm{CaCl}_{2}(\mathrm{pH} 8.2)$ to dilute urea to a final concentration of $2 \mathrm{M}$.

6. Add L-1-tosylamido-2 phenylethyl chloromethyl ketone (TPCK)-treated trypsin (50:1 substrate to enzyme mol ratio) to each sample and incubate at $37^{\circ} \mathrm{C}$ for $24 \mathrm{~h}$.

7. Quench the protein digestion by flash-freezing the sample in liquid nitrogen and store at $-80{ }^{\circ} \mathrm{C}$ until further processing.

\section{Sample Desalting}

1. Re-acidify the samples by adding formic acid (FA). Add enough FA to obtain a final concentration of $0.1 \%$. If samples are originally at $-80{ }^{\circ} \mathrm{C}$, thaw samples on ice before re-acidification.

2. De-salt the peptides using $C_{18}$ hydrophilic lipophilic balanced (HLB) 10 mg cartridges as previously described ${ }^{16}$.

3. Dry the samples by vacuum centrifugation ( 5 Torr, $45^{\circ} \mathrm{C}, 77 \times \mathrm{g}$ ) to a volume of $\sim 10 \mu \mathrm{L}$.

\section{Dimethylation Labeling (N-termini)}

1. Reconstitute peptides in $1 \%$ acetic acid $(0.25 \mu \mathrm{g} / \mu \mathrm{L})$ dissolved in high performance liquid chromatography (HPLC) or nano-pure grade water. Remove a $50 \mu \mathrm{g}$ portion to a new micro-centrifuge tube $(1.5 \mathrm{~mL})$ and store the remainder at $-80{ }^{\circ} \mathrm{C}$.

2. Add $8 \mu \mathrm{L}$ of $60 \mathrm{mM}(4 \%) \mathrm{CH}_{2} \mathrm{O}(37 \% \mathrm{wt} / \mathrm{v})$ or $60 \mathrm{mM}(4 \%){ }^{13} \mathrm{CD}_{2} \mathrm{O}(20 \% \mathrm{wt} / \mathrm{v})$ to the samples for labeling with light or heavy dimethylation, respectively. Typically, $4 \mu \mathrm{L}$ of reagent is added per $25 \mu \mathrm{g}$ of peptides (Steps $4.2,4.3,4.6$ ).

3. Add $8 \mu \mathrm{L}$ of $24 \mathrm{mM} \mathrm{NaBH}{ }_{3} \mathrm{CN}$ or $24 \mathrm{mM} \mathrm{NaBD}_{3} \mathrm{CN}$ to the samples labeled with light or heavy dimethylation, respectively.

4. Vortex and shake on a tube shaker for $\sim 10 \mathrm{~min}$ at room temperature.

5. Quench the reactions by adding $16 \mu \mathrm{L}$ of $1 \%$ ammonia $(\sim 28-30 \% \mathrm{v} / \mathrm{v})$ for $5 \mathrm{~min}$. Typically, $8 \mu \mathrm{L}$ of reagent is added per $25 \mu \mathrm{g}$ of peptides.

6. Re-acidify the reaction mixtures by adding $8 \mu \mathrm{L}$ of $5 \% \mathrm{FA}(98 \% \mathrm{v} / \mathrm{v})$.

7. Combine the light and heavy dimethylated peptides (Figure 1) to generate a total of six samples. See Table 1 for a description of sample tagging and pooling used for this study.

8. Perform sample desalting according to steps 3.2 and 3.3. 


\section{Isobaric Tagging (Lys residues)}

1. Reconstitute $100 \mu \mathrm{g}$ of dimethylated peptides $(1 \mu \mathrm{g} / \mu \mathrm{L})$ in $100 \mathrm{mM}$ tri-ethyl ammonium bicarbonate (TEAB) buffer (pH $~ 8.5)$.

2. Prepare isobaric reagents as stated in the manufacturer's protocol (see Table of Materials/Reagents).

3. Add solubilized isobaric reagents $(41 \mu \mathrm{L})$ to peptides and vortex for $\sim 10 \mathrm{~s}$. Shake the samples on a micro-centrifuge tube shaker for $\sim 1 \mathrm{~h}$. Quench the reactions with $8 \mu \mathrm{L}$ hydroxylamine $(10 \% \mathrm{w} / \mathrm{v})$ and incubate the samples for $15 \mathrm{~min}$ at room temperature.

4. Pool the six labeled samples into a single mixture and desalt (Steps $3.1-3.3$ ) or store at $-80^{\circ} \mathrm{C}$ until further use. NOTE: For improving proteome coverage and depth, a multi-dimensional separation strategy is suggested such as strong cation exchange $(\mathrm{SCX})$

\section{Strong Cation Exchange}

1. Perform SCX as per the manufacturer's protocol (see Materials Table).

1. Prepare $\sim 1 \mathrm{~mL}$ of ammonium formate solutions $(20 \mathrm{mM}, 40 \mathrm{mM}, 60 \mathrm{mM}, 80 \mathrm{mM}, 100 \mathrm{mM}, 150 \mathrm{mM}, 250 \mathrm{mM}, \& 500 \mathrm{mM})$ with $10 \%$ acetonitrile (ACN), $0.1 \% \mathrm{FA}(\mathrm{pH} 3)$.

2. Remove the red cap from the top of the pipette tip and tap the pipette tip with packing slurry to ensure that the packing material is towards the bottom of the pipette tip.

NOTE: Critical: Do not throw away the pipette tip until the end of the protocol.

3. Place the centrifuge adapter onto the top of a micro-centrifuge tube $(2 \mathrm{~mL})$ and secure the pipette tip inside.

4. Add $150 \mu \mathrm{L}$ of SCX reconstitution buffer to pipette tips.

5. Centrifuge the pipette tips for $6 \mathrm{~min}$ at room temperature $(4,000 \mathrm{xg})$. Repeat this step two more times and discard the waste.

6. Dissolve the peptides in $150 \mu \mathrm{L}$ of SCX reconstitution buffer. Ensure that the $\mathrm{pH}$ is 3 .

7. Add peptides to the pipette tips, centrifuge (see 6.1.5), and keep the eluent.

8. Transfer the filter and pipette to a new micro-centrifuge tube $(2 \mathrm{~mL})$ and add $150 \mu \mathrm{L}$ of $20 \mathrm{mM}$ ammonium formate solution. Centrifuge for 6 min at room temperature $(4,000 \mathrm{x} \mathrm{g})$ and keep the eluent.

9. Repeat step 6.1.8 an additional seven times, using successive concentrations (i.e. $40 \mathrm{mM}, 60 \mathrm{mM}, 80 \mathrm{mM}, 100 \mathrm{mM}, 150 \mathrm{mM}, 250 \mathrm{mM}$ and $500 \mathrm{mM}$ ) of ammonium formate.

2. Transfer the eight SCX fractions to micro-centrifuge tubes $(1.5 \mathrm{~mL})$ and concentrate them by using centrifugal evaporation (see step 3.3 ).

\section{Liquid Chromatography-Tandem Mass Spectrometry (LC-MS/MS) and MS $^{3}$}

1. Reconstitute the peptides in mass spectrometry grade water with $0.1 \% \mathrm{FA}$, filter, and place into an auto-sampler vial. Filter peptides as follows:

1. Add reconstituted peptides to a micro-centrifuge tube containing a $0.65 \mu \mathrm{m}$ filter (see Materials Table).

2. Centrifuge peptides at $12,000 \times \mathrm{g}$ for $1 \mathrm{~min}$. Remove filter, discard, and add filtered peptides into an auto-sampler vial.

2. Prepare the mobile phase buffers as follows: $3 \%(v / v) A C N$ with $0.1 \% F A(A)$ and $100 \% A C N$ with $0.1 \%$ FA (B).

3. Inject $6 \mu \mathrm{L}$ of each $\mathrm{SCX}$ fraction sample onto a trap column packed to $2 \mathrm{~cm}$ with $\mathrm{C}_{18}$ material $(5 \mu \mathrm{m}, 200 \AA$ pore size $)$. NOTE: Sample cleaning on the trap is as follows: $3 \mathrm{~min}, 0 \% \mathrm{~B} ; 3 \mu \mathrm{L} / \mathrm{min}$ using a 2D liquid chromatography system (see Materials Table).

4. Run the analytical separation method. Use a $75 \mu \mathrm{m}$ i.d. $\times 13.2 \mathrm{~cm}$ laser pulled-tip fused silica capillary column packed with $\mathrm{C}_{18}$ material $(5$ $\mu \mathrm{m}, 100 \AA$ ). The gradient is: 0-5 min, $10 \% \mathrm{~B} ; 5-40 \mathrm{~min}, 10-15 \% \mathrm{~B} ; 40-90 \mathrm{~min}, 15-25 \% \mathrm{~B} ; 90-115 \mathrm{~min}, 25-30 \% \mathrm{~B} ; 115-130 \mathrm{~min}, 30-60 \% \mathrm{~B}$; $130-135 \mathrm{~min}, 60-80 \% \mathrm{~B} ; 135-145 \mathrm{~min}, 80 \% \mathrm{~B}, 145-150 \mathrm{~min}, 80-10 \% \mathrm{~B} ; 150-180 \mathrm{~min}, 10 \% \mathrm{~B} ; 300 \mathrm{~nL} / \mathrm{min}, 180 \mathrm{~min}$.

5. Run the data acquisition for the mass spectrometer while the analytical separation method is running. NOTE: The parameters for the MS survey scan are as follows: $400-1,600 \mathrm{~m} / \mathrm{z}, 60,000$ resolution, automatic gain control (AGC) target $1 \times 10^{6}$ ions, maximum injection time $500 \mathrm{~ms}$. Parameters for CID-MS/MS are as follows: Top 1-7 or Top 8-14 data dependent acquisition (DDA), 3 $\mathrm{m} / \mathrm{z}$ isolation width, 500 minimum signal, $35 \%$ normalized collision energy (NCE), 0.25 activation q, $10 \mathrm{~ms}$ activation time, $3 \times 10^{4}$ AGC, 50 $\mathrm{ms}$ maximum injection time. Parameters for HCD-MS ${ }^{3}$ are as follows: 200 minimum signal, $4 \mathrm{~m} / \mathrm{z}$ isolation width, $60 \%$ NCE, 0.1 activation time, $3 \times 10^{5}$ AGC, $250 \mathrm{~ms}$ IT, 300-1,300 m/z MS/MS selection range, exclude un-fragmented parent ion.

6. Perform each analysis in duplicate for both the top 1-7 and top 8-14 runs.

NOTE: For samples run on a newer model of an instrument containing an orbitrap or a tribrid mass spectrometer, DDA parameters will vary and can be optimized to include more MS/MS and $\mathrm{MS}^{3}$ scans. Additionally, for tribrid instruments, synchronous precursor selection (SPS)$\mathrm{MS}^{3}$ should be employed.

\section{Data Analysis ${ }^{16}$}

1. Search the RAW files using protein analysis software against an appropriate database, such as mouse Uniprot. NOTE: It is important to create two workflows for each RAW file in order to search for light and heavy dimethylated peptides.

2. Search the RAW files using the following parameters: trypsin with two missed cleavages, peptide mass range 300-6,000 Da, $15 \mathrm{ppm}$ parent mass tolerance, $1 \mathrm{Da}$ fragmentation tolerance. Static modifications: light dimethylation $(+28.031$, peptide $\mathrm{N}$-terminus) or heavy dimethylation (+36.076 Da, peptide N-terminus), carbamidomethyl (+57.021 Da, C).

NOTE: Sometimes the heavy dimethylated peak is $\sim 7 \mathrm{Da}(+35.069 \mathrm{Da}$, peptide N-terminus) is from the light dimethylated peak and thus should be incorporated into the search workflow for heavy dimethylated peptides. Dynamic modifications: oxidation (+15.995 Da, M), 6plex isobaric tag (229.163 Da, K). Include a decoy database search to yield false discovery rates (e.g. 1 and $5 \%$ ) and a quantitation node to search for the reporter ion intensities of isobaric tags.

3. Statistics 
1. Export data into an electronic spreadsheet program in order to normalize protein reporter ion values. For each protein, divide either the median reporter ion ratios or raw reporter ion intensities by the median ratio of the internal standard or raw reporter ion intensities of the internal standard, respectively. Use the appropriate statistical software such as an electronic spreadsheet program, Perseus, or R to determine significant changes among sample conditions.

\section{Representative Results}

CPILOT uses amine-based chemistry to chemically label peptides at the N-terminus and lysine residues and enhances sample multiplexing capabilities. Figure 2 shows representative MS data that is obtained from a 12-plex cPILOT analysis of brain, heart, and liver tissues from an Alzheimer's disease mouse model and wild-type controls. As shown in Table 1, two biological replicates for the Alzheimer's disease and wildtype mice are included in this 12-plex analysis. Figure 2A shows a doubly-charged peak pair that is separated by $\mathrm{m} / \mathrm{z}$ spacing of 4 indicating a single dimethyl group was incorporated into the peptide. Both the light and heavy dimethylated peaks in this pair are independently isolated and fragmented with CID. The MS/MS data for each of the dimethylated peptides is shown in Figures 2B and C. Search results indicate this pair of peaks belongs to the T(dimethyl)ELNYFAK(isobaric-tag ${ }^{6}$ ) peptide of the protein phosphoglycerate kinase 1. The most intense fragment ion is $\mathrm{y}^{3+}$ which is similar for both the light and heavy dimethylated peaks. These peaks are isolated further for HCD-MS ${ }^{3}$ and the reporter ions $(\mathrm{m} /$ z 126-131) are observed as shown in Figures 2D and 2E. Both sets of $\mathrm{MS}^{3}$ spectra are necessary to get information about the 12 samples. In this example, the reporter ion ratios (AD/WT) (Alzheimer's disease/Wild-type control) for brain, liver, and heart tissues are similar across the two biological replicates. The fold-change values for each comparison suggest that phosphoglycerate kinase 1 levels in brain and heart are higher in AD mice, whereas in liver the levels are lower. 


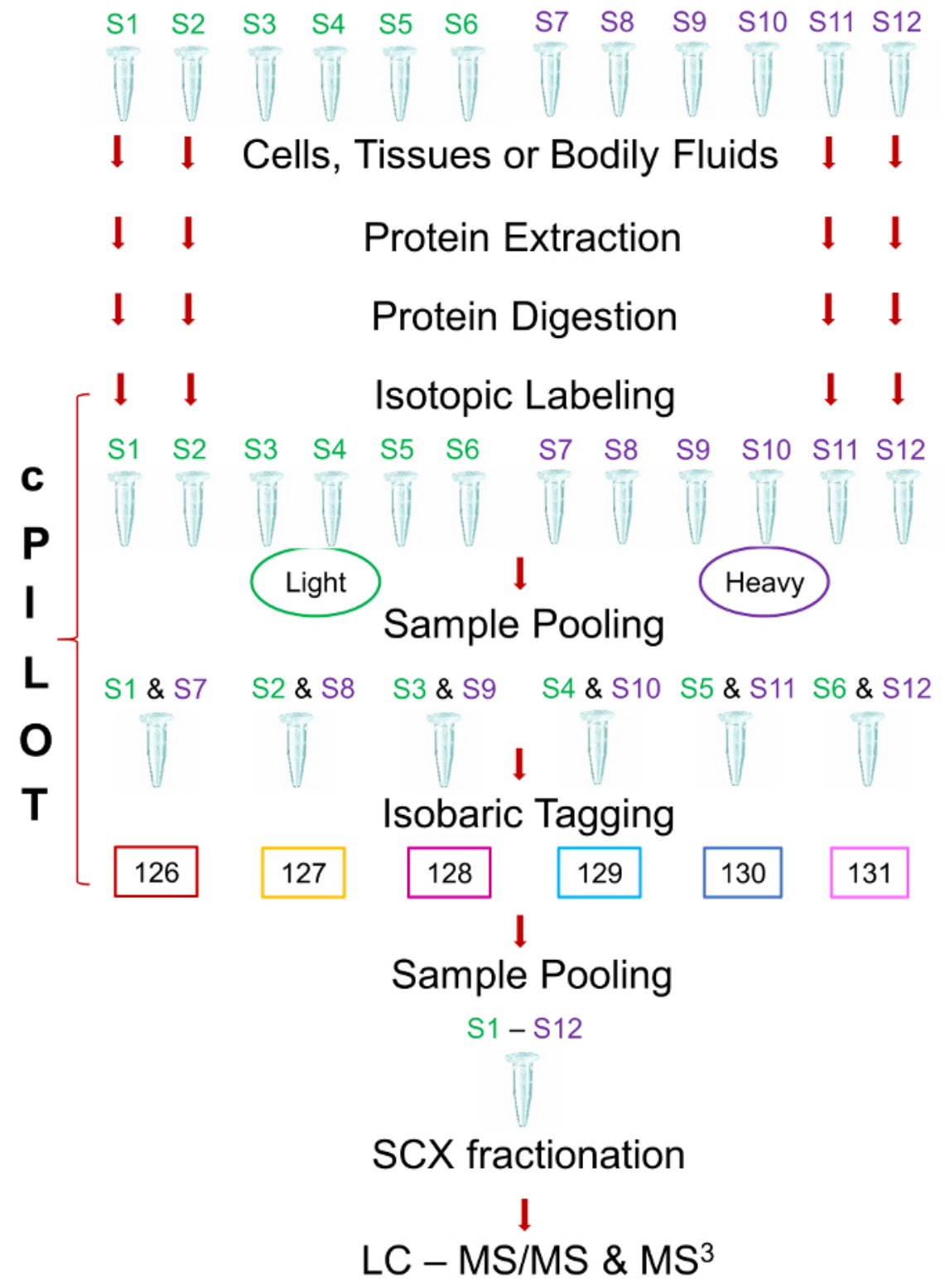

Figure 1: Proteomics workflow using cPILOT. As an example, this workflow outlines the analysis of 12 individual samples. Proteins from tissues, cells, or bodily fluids are extracted and a suitable protein standard (e.g. bovine alpha-casein) is added. Proteins are digested using trypsin. Peptides are labeled at the N-terminus by using light or heavy dimethylation $(\mathrm{pH} \sim 2.5$ ) and at lysine residues by using TMT6-plex (pH 8.5). Labeled peptides are pooled into a single mixture and subject to SCX RP-LC-MS/MS and MS ${ }^{3}$. Please click here to view a larger version of this figure. 

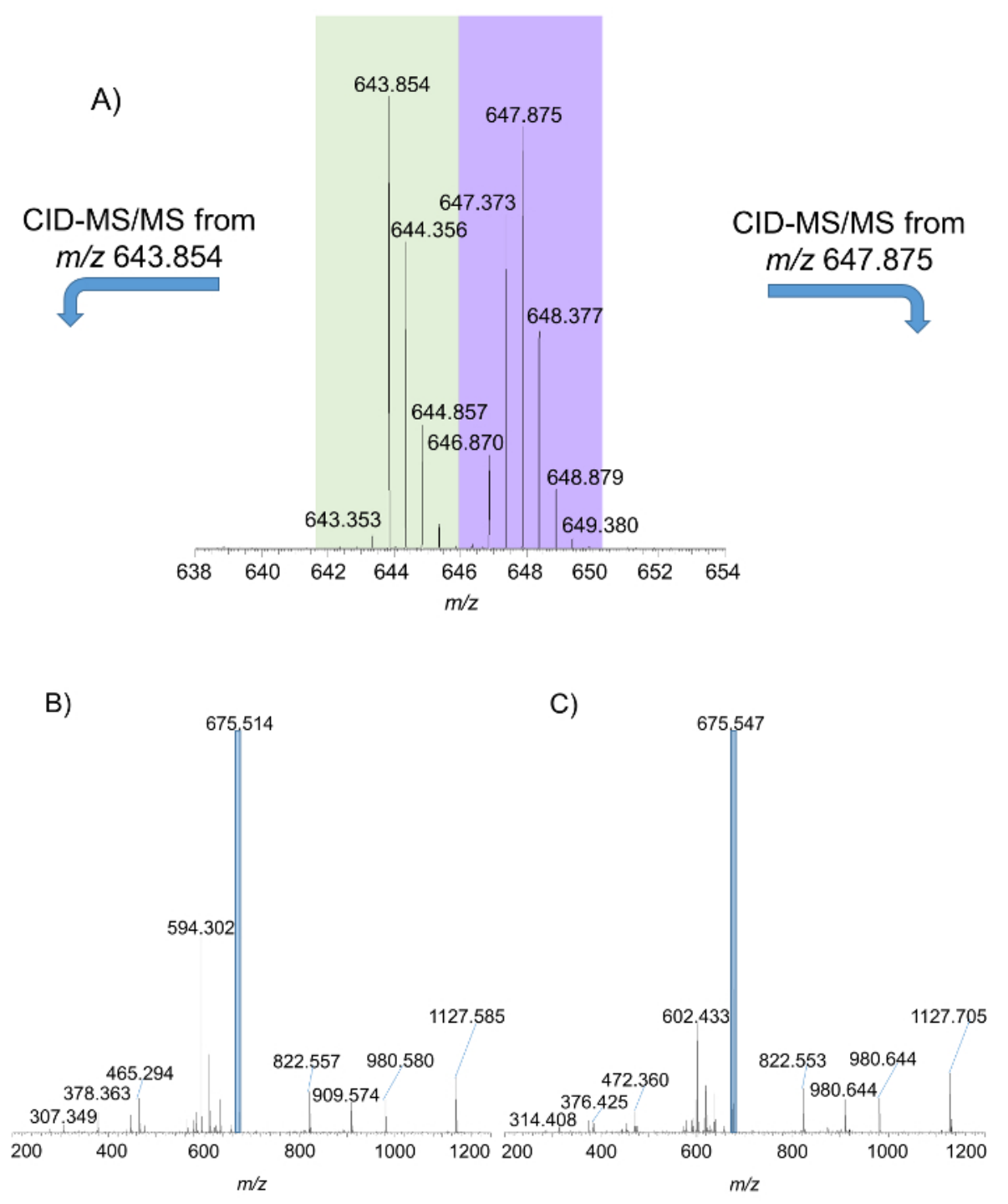

D)

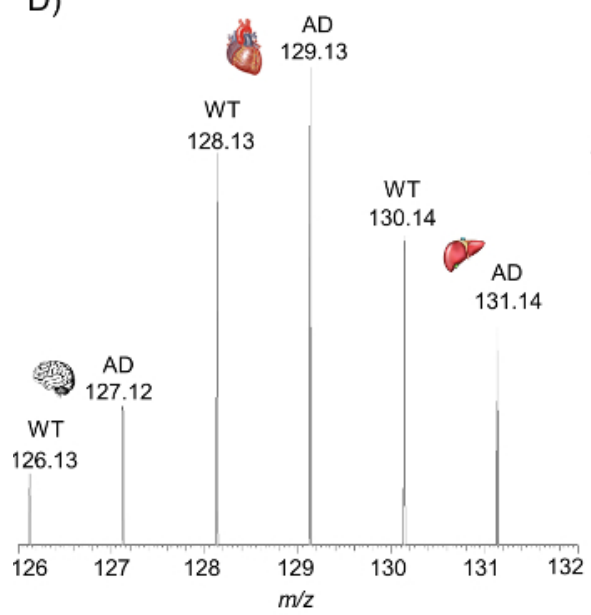

E)

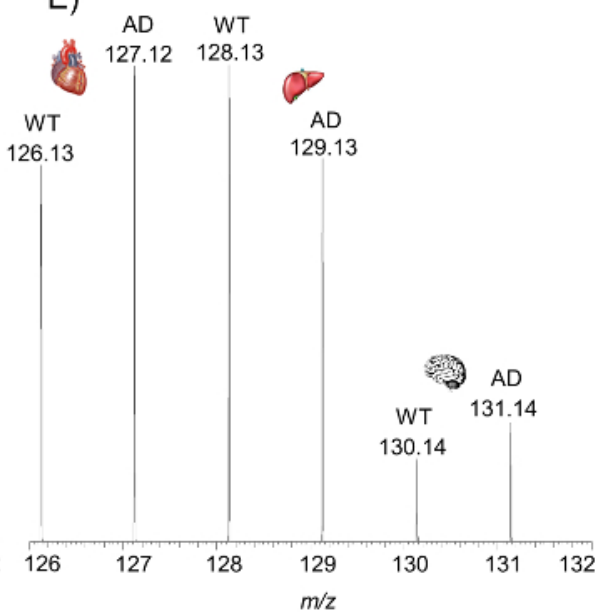

Figure 2: Example cPILOT data of peptides from brain, heart, and liver tissues of an Alzheimer's disease mouse model and wild-type controls. Precursor data (A) shows light and heavy dimethylated peptides, represented by the peaks at $m / z 643.854$ and 647.875 . These peptides were selected, isolated, and fragmented, thus generating CID-MS/MS spectra (B and $\mathbf{C}$ ), which provided peptide identification. An 
additional selection, isolation, and fragmentation of the most intense fragment ion of the light and heavy dimethylated peptides at the MS/ MS stage generated HCD-MS ${ }^{3}$ spectra (D and E), respectively. The peptide sequence is $T$ (dimethyl)ELNYFAK(isobaric-tag ${ }^{6}$ ) and belongs to phosphoglycerate kinase 1 . Please click here to view a larger version of this figure.

\begin{tabular}{|c|c|c|c|c|c|c|}
\hline & \multicolumn{6}{|c|}{ Isobaric Reagent } \\
\hline & 126 & 127 & 128 & 129 & 130 & 131 \\
\hline \multirow[t]{2}{*}{ Light Dimethylation } & $\mathrm{WT}^{\mathrm{a}}$ & $A D^{b}$ & WT & $A D$ & WT & $A D$ \\
\hline & \multicolumn{2}{|l|}{ brain } & \multicolumn{2}{|c|}{ heart } & \multicolumn{2}{|l|}{ liver } \\
\hline \multirow{2}{*}{$\begin{array}{l}\text { Heavy } \\
\text { Dimethylation }\end{array}$} & WT & $A D$ & WT & $A D$ & WT & $A D$ \\
\hline & \multicolumn{2}{|c|}{ heart } & \multicolumn{2}{|l|}{ liver } & \multicolumn{2}{|c|}{ brain } \\
\hline
\end{tabular}

Table 1: cPILOT grouping of AD and WT Brain, Heart, and Liver Tissues.

\section{Discussion}

CPILOT allows for the simultaneous measurement of more than 12 unique samples. In order to ensure successful tagging at both the $\mathrm{N}$-terminus and lysine residues of peptides, it is imperative to have the correct $\mathrm{pH}$ for each set of reactions and to perform the dimethylation reaction first for peptide labeling. Selective dimethylation at the $\mathrm{N}$-terminus is performed by having a pH at $\sim 2.5( \pm 0.2)$. This is achieved by exploiting the differences of the pKA's of the amino groups on lysine and the N-terminus. At pH 2.5, lysine is inactive (pKA 10.5); however, if the pH is mildly acidic (i.e. $\mathrm{pH} \mathrm{5-7)}$ ) or basic, both the N-termini and lysine residues will be dimethylated. In addition, if isobaric tagging is performed first at a low $\mathrm{pH}$, the $\mathrm{N}$-termini will have the isobaric label and lysine residues will be dimethylated. This may result in less fragments being selected for $\mathrm{MS}^{3}$ as b-ions would need to be selected. The relative costs of the dimethylation reactions are inexpensive compared to the commercial isobaric reagents. While one can use an entire isobaric reagent vial per $100 \mu \mathrm{g}$, we have also had success using half of the reagent vial per $100 \mu \mathrm{g}$ with comparable labeling efficiency. This helps to significantly reduce the costs for individual cPILOT experiments and allows more samples to be analyzed. Also it is important to note that other isobaric tagging reagents can be used in place of the isobaric reagent used in this protocol as we have previously demonstrated ${ }^{14}$. To ensure high labeling and tagging efficiencies, it is important to add reagents to samples quickly. This will allow for samples to have approximately the same reaction time. For quantitative purposes, each sample should be treated identically especially prior to pooling samples at the dimethylation and isobaric labeling steps. Finally, it should be noted that working with many samples simultaneously in the initial steps requires careful user skill and attention to sample handling.

The most ideal scenario is to obtain reporter ions for every protein in the mixture across the 12 samples. However, this is not the case for a large number of proteins. The number of peptides detected with quantitation information for cPILOT and other enhanced multiplexing approaching depends on several factors including sample type, sample fractionation and processing steps, MS data acquisition methods, and instrument type. Although both dimethylation and isobaric tagging steps have a high peptide labeling efficiency of $\sim 95-99 \%$, there is still $\sim 20 \%$ of the MS $^{3}$ data which will not contain reporter ion information. This is due in part to using trypsin which generates arginine-terminated peptides that result in no incorporation of isobaric reagent. This can be solved using other enzymes, such as LysC, with a potential tradeoff in protein identifications ${ }^{25}$. Also for heavy dimethylated peptides, the peak selected for fragmentation can be the M or M-1 peak, which have different intensities and will affect the reporter ion intensities observed in the $\mathrm{MS}^{3}$ stage. Thus there may be some low intensity reporter ions that are not detected for some samples. Such situations are often commonly observed for low intensity MS/MS fragments that are isolated for MS ${ }^{3}$ steps. A great solution to this issue has been incorporated into tribrid mass spectrometers, which, instead of using single-notch selection for $\mathrm{MS}^{3}$, use multi-notch or multiple MS/MS fragments ${ }^{26}$.

There is a lot of versatility in the cPILOT approach especially with regards to the number of samples that can be compared in a single analysis (i.e., up to 20 with commercial isobaric reagents) and the types of tissues used. We demonstrated that it is easy to obtain accurate quantitative information from brain, heart, and liver tissues in the same analysis in the context of a disease. This method, similarly to other multiplexing methods, allows for the analysis of multiple samples at once, and is applicable to samples originating from cells, tissues, bodily fluids, or whole organisms. In addition, CPILOT labeled peptides are able to be analyzed using either a low ${ }^{24}$ or high resolution $(60,000)$ instrument. In comparison, obtaining successful measurement using other multiplexing methods may be limited to a specific type of sample (i.e. cells $)^{18}$, may require an instrument with very high resolution ${ }^{20}$, or access to stable isotope labels. cPILOT is great for users interested in disease understanding, biomarker discovery, response to a drug or therapeutic intervention, or longitudinal changes across many time points. Furthermore, for large shotgun proteomics analyses of clinical samples (where $\mathrm{N}$ is large, hundreds to thousands), cPILOT may also be suitable to help reduce experimental costs and time. In the future, cPILOT will be expanded to multiplex a larger number of samples by using existing and novel isotopic and isobaric labels.

\section{Disclosures}

The authors have no competing interests.

\section{Acknowledgements}

The authors acknowledge the University of Pittsburgh Start-up Funds and NIH, NIGMS R01 grant (GM 117191-01) to RASR. 


\section{References}

1. Ong, S.-E. et al. Stable Isotope Labeling by Amino Acids in Cell Culture, SILAC, as a Simple and Accurate Approach to Expression Proteomics. Mol Cell Proteomics. 1 (5), 376-386 (2002).

2. Koehler, C. J., Arntzen, M. Ø., de Souza, G. A., \& Thiede, B. An Approach for Triplex-Isobaric Peptide Termini Labeling (Triplex-IPTL). Anal. Chem. 85 (4), 2478-2485 (2013).

3. Langen, H. F., M.; Evers, S.; Wipf, B.; Berndt, P. From Genome to Proteome, 3rd Siena 2D Electrophoresis Meeting. Wiley-VCH, Weinheim, Germany, Siena. (1998).

4. Yao, X., Freas, A., Ramirez, J., Demirev, P. A., \& Fenselau, C. Proteolytic 180 Labeling for Comparative Proteomics: Model Studies with Two Serotypes of Adenovirus. Anal. Chem. 73 (13), 2836-2842 (2001).

5. Reynolds, K. J., Yao, X., \& Fenselau, C. Proteolytic 180 Labeling for Comparative Proteomics: Evaluation of Endoprotease Glu-C as the Catalytic Agent. J. Proteome Res. 1 (1), 27-33 (2002).

6. Gygi, S. P. et al. Quantitative analysis of complex protein mixtures using isotope-coded affinity tags. Nat Biotech. 17 (10), $994-999$ (1999).

7. Schmidt, A., Kellermann, J., \& Lottspeich, F. A novel strategy for quantitative proteomics using isotope-coded protein labels. PROTEOMICS. 5 (1), 4-15 (2005).

8. Thompson, A. et al. Tandem Mass Tags: A Novel Quantification Strategy for Comparative Analysis of Complex Protein Mixtures by MS/MS. Anal. Chem. 75 (8), 1895-1904 (2003).

9. Ross, P. L. et al. Multiplexed Protein Quantitation in Saccharomyces cerevisiae Using Amine-reactive Isobaric Tagging Reagents. Mol Cell Proteomics. 3 (12), 1154-1169 (2004).

10. Xiang, F., Ye, H., Chen, R., Fu, Q., \& Li, L. N,N-Dimethyl Leucines as Novel Isobaric Tandem Mass Tags for Quantitative Proteomics and Peptidomics. Anal. Chem. 82 (7), 2817-2825 (2010).

11. Ting, L., Rad, R., Gygi, S. P., \& Haas, W. MS3 eliminates ratio distortion in isobaric multiplexed quantitative proteomics. Nat Meth. 8 (11), 937-940 10.1038/nmeth.1714 (2011).

12. McAlister, G. C. et al. Increasing the Multiplexing Capacity of TMTs Using Reporter Ion Isotopologues with Isobaric Masses. Anal. Chem. 84 (17), 7469-7478 (2012).

13. Frost, D. C., Greer, T., \& Li, L. High-Resolution Enabled 12-Plex DiLeu Isobaric Tags for Quantitative Proteomics. Anal. Chem. 87 (3), 1646-1654 (2015).

14. Robinson, R. A. S., \& Evans, A. R. Enhanced Sample Multiplexing for Nitrotyrosine-Modified Proteins Using Combined Precursor Isotopic Labeling and Isobaric Tagging. Anal. Chem. 84 (11), 4677-4686 (2012).

15. Evans, A. R., \& Robinson, R. A. S. Global combined precursor isotopic labeling and isobaric tagging (cPILOT) approach with selective MS3 acquisition. PROTEOMICS. 13 (22), 3267-3272 (2013).

16. Evans, A. R., Gu, L., Guerrero, R., \& Robinson, R. A. S. Global cPILOT analysis of the APP/PS-1 mouse liver proteome. PROTEOMICS Clin Appl. 9 (9-10), 872-884 (2015).

17. Gu, L., Evans, A. R., \& Robinson, R. A. S. Sample Multiplexing with Cysteine-Selective Approaches: cysDML and cPILOT. J. Am. Soc Mass Spectrom. 26 (4), 615-630 (2015).

18. Dephoure, N., \& Gygi, S. P. Hyperplexing: A Method for Higher-Order Multiplexed Quantitative Proteomics Provides a Map of the Dynamic Response to Rapamycin in Yeast. Sci Signal. 5 (217), rs2-rs2 (2012).

19. Hebert, A. S. et al. Neutron-encoded mass signatures for multiplexed proteome quantification. Nat Meth. 10 (4), $332-334$ (2013).

20. Merrill, A. E. et al. NeuCode Labels for Relative Protein Quantification. Mol Cell Proteomics. 13 (9), 2503-2512 (2014).

21. Braun, C. R. et al. Generation of Multiple Reporter lons from a Single Isobaric Reagent Increases Multiplexing Capacity for Quantitative Proteomics. Analytical Chemistry. 87 (19), 9855-9863 (2015).

22. Everley, R. A., Kunz, R. C., McAllister, F. E., \& Gygi, S. P. Increasing Throughput in Targeted Proteomics Assays: 54-Plex Quantitation in a Single Mass Spectrometry Run. Anal. Chem. 85 (11), 5340-5346 (2013).

23. Gu, L., \& Robinson, R. A. S. High-throughput endogenous measurement of S-nitrosylation in Alzheimer's disease using oxidized cysteineselective cPILOT. Analyst. 141 (12), 3904-3915 (2016).

24. Cao, Z., Evans, A. R., \& Robinson, R. A. S. MS3-based quantitative proteomics using pulsed-Q dissociation. Rapid Commun Mass Spectrom. 29 (11), 1025-1030 (2015).

25. Swaney, D. L., Wenger, C. D., \& Coon, J. J. Value of Using Multiple Proteases for Large-Scale Mass Spectrometry-Based Proteomics. J. Proteome Res. 9 (3), 1323-1329 (2010).

26. McAlister, G. C. et al. MultiNotch MS3 Enables Accurate, Sensitive, and Multiplexed Detection of Differential Expression across Cancer Cell Line Proteomes. Anal. Chem. 86 (14), 7150-7158 (2014). 